\title{
Anti-cancer and Biotherapeutic Potentials of Probiotic Bacteria
}

\section{Zubaida Hassan*}

Department of Microbiology, School of Life Sciences, Modibbo Adama University of Technology, Yola, Nigeria

\begin{abstract}
Current standard cancer drugs and various synthetic agents exhibit high toxic activities against cancer cells, but doubts have been raised as to their long term stability and safety. Generally, these synthetic agents are relatively expensive; this makes them not affordable to many people. Although most probiotic anticancer therapies are in preclinical development phase, due to their low efficacy and poor selectivity, gradual replacement of current cancer drugs and other synthetic agents with appropriate biotherapeutic substances is proposed to overcome the challenges associated with the use of these synthetic agents. These probiotics can have an effect on other aspects of human health and hence make life worth living during and after cancer treatment. At present, most anticancer research regarding probiotic microbes focuses on Lactobacillus and Bifidobacteria, but other probiotics are involved. This review describes the properties of probiotic bacteria as potential biotherapeutics to supplement current standard anti-cancer therapies. The reader will gain an overview of different probiotics tested so far with respective bioassays used in probiotic anti-cancer drug discovery. Note, Not all therapies used generated an effective response in all patients and that use of probiotic therapies provides negligible if any, detrimental side effects.
\end{abstract}

Keywords: Probiotics; Biotherapeutic agents; Cytotoxicity assays; Cancer

\section{Introduction}

Cancer is a deadly disease with high clinical significance and is mostly diagnosed at a late stage. It is defined as uncontrolled cell growth [1], and as a disease caused by a deficiency in DNA repair [2]. The mechanisms that initiate cancer remain unclear. To date, chemotherapeutics are the standard drugs used in the clinical treatment of cancer. However, these compounds are accompanied with considerable and complicated lifethreatening side effects that most times gets worse than the tumour. As a protective alternative, recent research has focused on the biotherapeutic potentials of probiotic bacteria in cancer treatment. Supplementation with probiotics is proposed to improve quality of life during and after chemotherapy treatment. This is because these bacteria are generally regarded as safe, have long history of usage and affordable than chemotherapy.

For a long time, humans have relied on natural products and synthetic compounds as sources of medicine; these are mainly plant-based natural products [3-5]. Additionally, microbes and their derivatives possess pharmacological and/or biological ability that can enable them to be used in treating human diseases. Probiotic bacteria, for example, have been studied for their anticancer properties and have shown promising results [5-8]. Probiotics are defined as live microorganisms which, when taken in adequate amounts, can exert beneficial effects through their growth or other activities in the host.

The mechanisms by which cancer starts and develops remain unclear. Hence, while trying to fully understand its biology, it is necessary to find ways that can reduce the severity of the disease and improve patients' quality of life. Probiotic organisms prevent cancer through various mechanisms, among which are the secretion of soluble compounds during fermentation [9], triggering the immune response by natural killer cells [10], interfering with the synthesis of oestrogen in both normal and tumour-invaded breast tissues [11] and through competitive exclusion of pathogenic microbes in the intestine [12]. Others include reduced enterocyte apoptosis [13], modulation of inflammation [14], and maintenance of barrier function thus, suggesting that a probioticbased therapy could be an effective therapeutic strategy [15].

\section{Literature Review Criteria}

Information for this review was compiled by searching the PubMed database for published articles using search terms "probiotic", "biotherapeutics" and "lactic acid bacteria" + the terms "cancer" or "oncology". Full articles were obtained, and references were checked for additional material where necessary.

According to Compare and Nardone [16], chronic bacterial infection can induce inflammatory processes, especially in the gastrointestinal tract. For example, Helicobacter pylori infection is associated with both gastric cancer and mucosa-associated lymphoid tissue lymphoma. However, probiotic microbes have the ability to inhibit a wide range of pathogens, thereby, preventing the initiation of infection with bacteria that may lead to development of tumour. The polysaccharide fraction of heat killed cells of these probiotics has been found to inhibit cancer cell proliferation while producing less cytotoxic effects on normal cells [6]. In addition to the heat killed fractions of probiotics, we previously reported anti-breast cancer effects from live as well as cytoplasmic fractions of S. hominis and E. faecalis [17]. This is an indication that heat soluble polysaccharides may play a significant role in the anticancer effects of these bacteria [6].

\section{Effects of probiotic species on cancer in vitro}

With increase in cancer incidences coupled with adverse side effect of current therapies, researchers are focusing more on the use of probiotic bacteria as anticancer and antioxidant agents to be protective adjuncts against human diseases [5-8,17]. Although various synthetic agents exhibit even higher cytotoxic activities on most cancer than probiotics, people are concerned about the stability and safety of these

*Corresponding author: Zubaida Hassan, Department of Microbiology, Schoo of Life Sciences, Modibbo Adama University of Technology, Yola, Nigeria, Tel: +2347029273726; E-mail: zubaidahassan@mautech.edu.ng

Received January 05, 2019; Accepted January 19, 2019; Published January 21, 2019

Citation: Hassan Z (2019) Anti-cancer and Biotherapeutic Potentials of Probiotic Bacteria. J Cancer Sci Ther 11: 009-013. doi: 10.4172/1948-5956.1000575

Copyright: (c) 2019 Hassan Z. This is an open-access article distributed under the terms of the Creative Commons Attribution License, which permits unrestricted use, distribution, and reproduction in any medium, provided the original author and source are credited. 
synthetic substances in the long term. Generally, these synthetic agents are relatively more expensive [6]. Nami [5] reported that Enterococcus faecalis strains exert anti-proliferative effects on a breast cancer cell line, resulting in $41.27 \%$ viability, while having little effect on the viability (94.02\%) of normal human umbilical vein endothelial cells (HUVEC). Similarly, in our previous study, it was shown that E. faecalis and $S$. hominis inhibit up to $66.99 \% 31.09 \%$ viabilities in MCF-7 cells after coculture for $24 \mathrm{~h}$ with negligible effects of $>90 \%$ viabilities on their nonmalignant control, MCF-10 cells [17].

Lactobacillus acidophilus and Lactobacillus casei excrete antitumour substances which have been suggested to be components of the bacterial cell wall [18]. Freeze dried Lactobacillus acidophilus La-05 and Lactobacillus casei spp. paracasei Lc-01 showed anti-proliferative effects against a breast cancer cell line (MCF7), even in the absence of live organisms. The secretion of soluble compounds during fermentation was thought to be responsible for these beneficial effects [9]. Hirayama and Rafter [19] also reported the growth inhibition of MCF7 cells by compounds produced by $L$. acidophilus and $L$. casei. In addition, Lactobacillus acidophilus 606 also inhibits human pancreatic cancer cell line (PANC-1) proliferation by the production of soluble polysaccharides.

\section{Effects of probiotic species on cancer and inflammation in vivo}

Various experimental animal models have been used to evaluate the anti-cancer properties of different probiotics. Lactobacillus acidophilus for example, has been shown to induce IL-12 production in $\mathrm{BALB} / \mathrm{c}$ mice; this interleukin can then trigger immune responses by natural killer cells [6]. Lactobacillus casei spp. casei ATCC 39392 was administered orally to 6-8-week-old female BALB/c mice for two weeks before transplanting tumour cells, after which the probiotic bacteria were continually administered for three weeks with three-day intervals between the weeks. The results from this study showed an increase in IL-12, IFN- $\gamma$ and natural killer cells [20]. It is well-known that IL-12, IFN- $\gamma$ and natural killer cells are modulators of the immune system, vital players in both innate and adaptive immune responses. With this, it can be proposed that probiotics therapy can be long live through generation of memory cells from adaptive immunity.

Lactobacilli were shown to delay the development of tumours in both normal and tumour-invaded breast tissues. De Moreno De LeBlanc [11] reported that Lactobacillus helveticus R389 is able to delay the development of tumours. This was related to peptide production by this organism, which decreased the expression of IL- 6 and increased the expression of IL-10, and also induced apoptosis in tumour cells. IL-6 is a cytokine that stimulates aromatase activity, an enzyme involved in the synthesis of oestrogen in both normal and tumour-invaded breast tissue. Oestrogen is hypothesized to influence breast cancer mediated through estrogen receptor $\alpha$ as well as estradiol, but the mechanism is poorly understood [21]. IL-10 on the other hand, is a pleiotropic anti-inflammatory cytokine [22]. Although increase expression of IL10 is not a favourable prognostic value due to its immunosuppressive nature, but its antiangiogenic functions [23] is vital in checking tumour growth. IL-10 inhibits tumorigenesis via down-regulation of VEGF, IL-1b, TNF- $\alpha$, IL- 6 , and MMP-9 through translocation of NF- $\kappa B$ pathway [23]. Breast cancer susceptibility may be attributed to IL-10 gene polymorphisms.

In experimental dog models of cyclophosphamide-induced neutropenia, the duration of the condition was shortened and augmentation of leukocyte reconstituting capacity was enhanced after the administration of a heat killed strain E. faecalis [24]. In another study, milk fermented with B. lactis, L. lactis, S. thermophilus, and L. bulgaricus was found to improve inflammation in a mouse model of colitis, increase butyrate-producing bacteria, and decrease the number of Enterobacteriaceae strains which are capable of inducing colonic inflammation [25]. It has been shown that Lactobacilli and Bifidobacteria are able to increase the probiotic concentration and significantly decrease putrefactive microorganisms such as coliforms in the faeces of animals $[26,27]$. These putrefactive microorganisms are associated with the synthesis of putative carcinogens in the colon $[28,29]$ and such microbes are linked to decrease in tumorigenesis in the IL-10 knockout mice model [29].

Beyond animal studies, few researchers have discussed the biotherapeutic effects of probiotics in humans. The consumption of probiotic fermented dairy products has a long history in cancer research. In the year 1977, an epidemiological study conducted by Malhotra, in Finland revealed that, despite a high fat intake, the incidence of colon cancer was low compared to other countries [30]. A reduction in procarcinogenic elements such as mutagens found in a western, meatrich diet was observed in the urine and faeces of healthy volunteers who consumed Lactobacilli. In most cases, mutagens excreted in the urine and faces are inversely proportional to the lactobacilli secreted [31]. Therefore, it was hypothesised that the Lactobacilli consumed were responsible for this effect.

In a case control study by Veer [32] conducted in the Netherlands, a country that has a high incidence of breast cancer, suggested the protective ability of fermented milk and Gouda cheese on the risk of this cancer. However, no statistically significant relationship was found between the consumption of non-fermented milk and breast cancer. According to Toi [33], daily consumption of Lactobacillus casei Shirota has a significant, inverse association with the early occurrence of breast cancer, irrespective of menopausal status. In population-based case-control studies, an inverse association has been documented for colorectal cancer and the consumption of yoghurt or other cultured milk products [34,35]. An inverse relationship has also been demonstrated between breast cancer in women and the consumption of yoghurt and other fermented milk products [16].

Comparing the stool bacteria of colorectal cancer patients and that of healthy individuals, there was a significant decrease in Bifidobacteria and Ruminococcus bromii $[12,36]$. These bacteria were found in the mucosa-adherent microbiota in the cancer patients at a lower rate than in healthy individuals [37]. Similarly, a randomised, double-blind and placebocontrolled trial with 26 healthy adults conducted by Johansson [38] demonstrated that the consumption of Lactobacillus plantarum fermented rose-hip drink for a period of three weeks significantly increased faecal probiotics and decreased pathogenic sulphite-reducing clostridia. As in animal studies, human studies have also shown that Lactobacillus acidophilus is capable of decreasing faecal putrefactive flora while increasing commensal lactobacilli [39]. These putrefactive microorganisms are associated with the synthesis of putative carcinogens in the colon $[28,29]$.

\section{Effects of cell free probiotic supernatants on cancer}

Live probiotic microbes as well as their heat killed cells, secreted metabolites and cytoplasmic fractions exert anticancer properties. Lactoglobulin and $\alpha$-lactalbumin have been reported to be the two major proteins that play important roles in the anticancer effects of lactobacillus. Lactoglobulin is a good source of sulphur amino acids and has immune modulatory effects, while $\alpha$-lactalbumin forms complexes 
with oleic acid, which are lethal to tumour cells [40]. Biotherapeutic efficacy assessments of $E$. faecalis secreted metabolites revealed apoptotic effects on human breast, colon, cervical, and gastric cancer cell lines while producing negligible cytotoxic effects on HUVEC (normal cell line). This selective effect is mainly associated with their ability to recognise proteins secreted by different cancer cells [5].

Heat killed cells and cytoplasmic fractions of eight different species of Lactobacillus and four species of Bifidobacteria were studied by Liu and Pan [8] against breast and colon cancer cell lines. They reported strong anti-proliferative and antioxidant effects of these microbes. Heat killed cells of these bacteria were able to reduce the viability of human breast adenocarcinoma cells to $46.3 \%$ with no inhibitory effects on the intestinal 407 cell line. We also observed similar effect when breast cancer cells (MCF-7) were cocultured with cytoplasmic fractions of bacteria in for $24 \mathrm{~h}$, we recorded $31.09 \%$ viabilities with negligible effects of $>90 \%$ viabilities on their non-malignant control, MCF-10 cells [17].

Metabolites extracted from Lactobacillus sp. used in yoghurt fermentation have been shown to have potential roles in the inhibition of growth and induction of apoptosis in human tongue squamous carcinoma (CAL-27) cells in vitro [40]. The metabolites, from Lactobacillus sp., contain a number of peptides, amino acids, short chain fatty acids, lactic acid, butyric acid, and certain other chemicals, which may play important roles in its anticancer activities. These metabolites induced cytotoxic effects in CAL-27 cells of early apoptotic by $12.8 \%$, while the percentage of the late cells was up to $69.48 \%$ [40]. Heat killed cells of Lactobacillus casei ATCC 393 and Lactobacillus acidophilus 606 have been reported to have inhibitory effects against cancer cells [6] by the production of soluble polysaccharides, which inhibit the proliferation of HT29 and HeLa cancer cells.

\section{Effects of probiotic species on side effects of cancer chemotherapy}

It is well-known that chemotherapy is at present is the standard treatment for cancer. However, it may not be the ideal therapy as it is associated with complicated side effects on patients. These side effects include chemotherapy-induced fatigue, gastrointestinal toxicity, haematological toxicity, alopecia, as well as cell and organ toxicity [41]. Chemotherapy is also associated with peripheral neuropathy and dysphonic syndrome [42]. In most cases, the side effects associated with these drugs are often of more concern than the progression of the tumour. For example, oxaliplatin is a promising drug routinely used in the treatment of human cancers such as colorectal, breast, ovarian, genitourinary, head/neck and gastroesophageal. However, it is associated with peripheral neuropathy [42].

Another widely used chemotherapy drug in treating variety of cancers is 5-fluorouracil (5-FU). Its administration also results in severe side effects such as arrhythmias, silent myocardial ischemia, angina, congestive heart failure, myocardial infarction, cardiogenic shock, and sudden death [43], intestinal and oral mucositis [15]. Those diagnosed with intestinal mucositis may suffer from nausea, vomiting, dyspepsia, dysphagia, and diarrhea [44]. These often leads to infection and malnourishment [45]. In many cases, the treatment must be ceased until the patient recovers from these severe effects [15]. While 5-FU compromise individual's immune system through these side effects, probiotics reduce risk of infection thus, renders them ideal for use in a disorder such as mucositis [15].

Currently, some probiotics are successful in the treatment of mucositis induced by 5-FU. For example, Lactobacillus fermentum BR11 and Streptococcus thermophilus TH-4 improved the histological deficits caused by 5 -FU $[46,47]$. Lactobacillus acidophilus combat 5-FU-induced changes in gastrointestinal motility, enhancing intestinal transit and gastric emptying and decreasing retention in the distal bowel segment thus normalizes bowel function by reducing inflammation associated with the chemotherapy [48].

Lactobacillus acidophilus is used for the treatment and prevention of gastrointestinal disorders associated with diarrhea of varying etiology [49] such as intestinal mucositis caused by the cytotoxicity of chemotherapy and radiotherapy used in cancer treatment [48]. In a clinical trial, Lactobacillus acidophilus associated with B. bifidum was satisfactory for diarrhea prophylaxis during pelvic radiation therapy with concomitant cisplatin. Acute inflammatory changes might play an important role in the pathogenesis of these symptoms [50].

Although, many probiotic anticancer therapies are yet to be tested in clinical trials, because of their low efficacy, they are associated with long-term stability and safety. Biotherapeutics may decrease the risk and severity of chemotherapy-related toxicity and side effects associated with treatment, as these organisms have the ability to enhance homeostasis in patients. Probiotics have been used for the prevention and treatment of chemotherapy-induced side effects such as infectious complications and diarrhoea [51]. The development of bowel problems often leads to infections in cancer patients; the use of antibiotics is such cases can lead to the dissemination of antibiotic resistance due to immunosuppression from chemotherapy. Thus, probiotics have been used in preventing bowel colonisation by pathogenic microorganisms through competitive inhibition [51].

While anticancer chemotherapeutics such as 5-FU cause diarrhoea in patients due to incomplete absorption of the drugs or the overuse of antibiotics [51], biotherapeutics are known to prevent this effect $[47,52]$ and so the use of probiotics is recommended in cancer chemotherapeutic treatment. Many of the current cancer therapeutic agents are limited in their use due of their toxic effects on normal cells and tissues, thus biotherapeutics may be an ideal supplementation with cancer chemotherapy and future alternative in cancer treatment and prevention.

\section{Potential mechanisms of probiotic action in cancer}

High degree of strain specificity is associated with mechanisms of probiotic action on cancer. The mechanisms by which probiotics reduce cancer may include the modulation of the immune response. This can differ according to the site where the tumour is present. Unlike in colon cancer, which is the most studied type of cancer where the consumption of fermented food product changes gut-associated immune cells and peritoneal macrophages, in the breast, immune cells changes are observed only when the target cell affects the mammary gland [11] Probiotics also act by potentiation of natural killer which mediates antitumor activity of a cell [53].

Lactic acid bacteria have been shown to prevent many types of cancer through the hydrolysis of glucuronides by $\beta$-glucoronidase, thereby liberating carcinogens [19], i.e., reducing glucuronidase, azoreductase, and nitroreductase, which can convert procarcinogens to carcinogens in the intestine $[54,55]$. These organisms prevent colon cancer by their production of azoxymethane, enhancing $\mathrm{O}^{6}$-methylguanine in colon mucosal DNA, and binding to and digesting free bioavailable toxins in the colon [54]. In addition, these organisms reduce DNA damage caused by chemical carcinogens [28]. Lactobacillus rhamnosus GG 
has demonstrated preventive ability on intestinal cells, preventing transepithelial electrical resistance (TEER) reduction and maintaining ZO-2 levels in Caco-2 cells treated with proinflammatory interferon$\delta$ [56] through the inhibition of proinflammatory tumor necrosis factor (TNF)- $\alpha$ expression, a cytokine which is also involved in the development of intestinal mucositis [57].

Anticancer probiotics also induce host innate defence mechanisms such as macrophage activation [58]. These microbes recognise and ligate pathogen-associated molecular patterns, dimerise tumour recognition receptors, especially toll-like receptors, and interfere with the transmission of intracellular signals of inflammation and tissue regeneration adaptor proteins [18]. Toll-like receptors are likely candidates to mediate the effects of the innate immune response on tumorigenesis [16]. The molecular mechanisms often associated with the anticancer effects of probiotics includes cytochrome P450 blockade, a reduction in carcinogen generation, downregulation of Ras-p21 expression, increased cell differentiation, inhibition of COX-2 upregulation, inhibition of nitric oxide synthase, increased short-chain fatty acid production, and a reduction in intestinal $\mathrm{pH}$ which inhibits putrefactive bacteria [58]. These genes are crucial in the initiation and development of cancer.

As a mechanism of cancer death induced by Glossogyne tenuifolia [4], E. faecalis and S. hominis inhibit MCF-7 cells proliferation by altering G0/G1 cell cycle phase [17]. Cytoplasmic fractions of Lactococcus lactis $s p p$. were used to treat a colon cancer cell line by attacking G0/G1 cell cycle growth phase [1]. This phenomenon may simply be related to the ability of the bacteria to cause the overexpression of cyclin A and under expression of cyclin E proteins [1]. Progression of the cell cycle from $\mathrm{G}_{1}$ to $S$ phase in eukaryotic cells is controlled by cyclins $\mathrm{A}, \mathrm{D}$, and $\mathrm{E}$. These proteins are responsible for the activation of different $G_{1}$ phase kinases (CDK4/6 and CDK2) [59].

Overexpression of cyclin D1 can lead to shortening of the $G_{1}$ phase of the cell cycle and subsequently lead to phenotypic changes. Transcription of cyclin D1 is promoted by the kinase cascade via the Ras signalling pathway [60]. In the nucleus, cyclin D1 is phosphorylated and activated by cyclin activating kinase (CAK) to form cyclin D/CAK4 complexes. The main function of cyclin D/CAK4 in the $\mathrm{G}_{1}$ phase is to inactivate growth suppressor proteins such as retinoblastoma protein (pRB). Phosphorylated $\mathrm{pRB}$ is capable of driving quiescent cells into $\mathrm{S}$ phase [60].

\section{Discussion and Conclusion}

Following the toxicity concern of current cancer chemotherapy and uncertainly in terms of long-term stability and safety of other synthetic compounds, probiotics are becoming relevant in the fight against cancer. These organisms have been shown to delay tumour formation, inhibit cancer cells proliferation, burst healthy growth in normal cells, enhance homeostasis and thus help prevent life-threatening side effects that accompany current cancer treatment. In addition, long-term stability and safety of probiotics in human consumption have been documented. These effects were also represented in animal models. Although, most of the in vivo trials have had small sample sizes, and thus there is a substantial risk of bias. It is strongly recommended that large, properly designed clinical trials to be carried out to establish proper evidences of biotherapeutic properties of these organisms as anticancer treatments. Finally, the emerging relationship between probiotics and cancer opens an interesting field in cancer prevention and treatment.

\section{References}

1. Kim JY, Woo HJ, Kim YS, Kim KH, Lee HJ (2003) Cell cycle dysregulation induced by cytoplasm of Lactococcus lactis ssp. lactis in SNUC2A, a colon cancer cell line. Nutr Cancer 46: 197-201.

2. Shaheen M, Allen C, Nickoloff JA, Hromas R (2011) Synthetic lethality: Exploiting the addiction of cancer to DNA repair. Blood 117: 6074-6082.

3. Celep G, Yilmaz S, Coruh N (2012) Antioxidant capacity and cytotoxicity of aesculus hippocastanum on breast cancer MCF-7 cells. J Food Drug Anal 20: 692-698.

4. Hsu H, Wu Y, Chen L, Houng J (2009) Induction of apoptosis of A549 lung cancer cell line by dehydrocostus lactone isolated from glossogyne tenuifolia. $J$ Food Drug Anal 17: 107-115.

5. Nami Y, Abdullah N, Haghshenas B, Radiah D, Rosli R, et al. (2014) A newly isolated probiotic Enterococcus faecalis strain from vagina microbiota enhances apoptosis of human cancer cells. J Appl Microbiol 117: 498-508.

6. Choi SS, Kim Y, Han KS, You S, Oh S, et al. (2006) Effects of Lactobacillus strains on cancer cell proliferation and oxidative stress in vitro. Lett Appl Microbiol 42: 452-458.

7. Kim JE, Kim JY, Lee KW, Lee HJ (2007) Cancer chemopreventive effects of lactic acid bacteria. J Microbiol Biotechnol 17: 1227-1235.

8. 8. Liu CF, Pan TM (2010) In vitro effects of lactic acid bacteria on cancer cell viability and antioxidant activity. J Food Drug Anal 18: 77-86.

9. Biffi A, Coradini D, Larsen R, Riva L, Di Fronzo G (1997) Antiproliferative effect of fermented milk on the growth of a human breast cancer cell line. Nutr Cancer 28: 93-99.

10. Janghorbani M, Amini M, Willett WC, Mehdi Gouya M, Delavari A, et al (2007) First Nationwide survey of prevalence of overweight, underweight, and abdominal obesity in Iranian adults. Obesity 15: 2797-2808.

11. 11. De Moreno De LeBlanc A, Matar C, Leblanc N, Perdigon G (2005) Effect of milk fermented by Lactobacillus helveticus R389 on a murine. Breast Cancer Res 7: 477-486.

12. Chong ES (2014) A potential role of probiotics in colorectal cancer prevention Review of possible mechanisms of action. World J Microbiol Biotechnol 30: 351-374.

13. Khailova L, Mount Patrick SK, Arganbright KM, Halpern MD, Kinouchi T, et al (2010) Bifidobacterium bifidum reduces apoptosis in the intestinal epithelium in necrotizing enterocolitis. Am J Physiol Gastrointest Liver Physiol 299: G1118-G1127.

14. Philippe D, Favre L, Foata F, Adolfsson O, Perruisseau-Carrier G, et al. (2011) Bifidobacterium lactis attenuates onset of inflammation in a murine model of colitis. World J Gastroenterol 17: 459-469.

15. Prisciandaro LD, Geier MS, Chua AE, Butler RN, Cummins AG, et al. (2012) Probiotic factors partially prevent changes to caspases 3 and 7 activation and transepithelial electrical resistance in a model of 5-fluorouracil-induced epithelial cell damage. Support Care Cancer 20: 3205-3210.

16. Compare D, Nardone G (2011) Contribution of gut microbiota to colonic and extracolonic cancer development. Dig Dis 29: 554-561.

17. Hassan Z, Mustafa S, Rahim RA, Isa NM (2016) Anti-breast cancer effects of live, heat-killed and cytoplasmic fractions of Enterococcus faecalis and Staphylococcus hominis isolated from human breast milk. In Vitro Cell Dev Biol Anim 52: 337-348.

18. Bogdanov IG, Dalev PG, Gurevich AI, Kolosov MN, Malékova VP, et al. (1975) Antitumour glycopeptides from Lactobacillus bulgaricus cell wall. FEBS Lett 57: 259-261.

19. Hirayama K, Rafter J (2000) The role of probiotic bacteria in cancer prevention Microbes Infect 2: 681-686

20. Soltan Dallal MM, Yazdi MH, Holakuyee M, Hassan ZM, Abolhassani M, et al. (2012) Lactobacillus casei ssp. casei induced Th1 cytokine profile and natural killer cells activity in invasive ductal carcinoma bearing mice. Iran J Allergy Asthma Immunol 11: 183-189.

21. Yue W, Wang JP, Li Y, Fan P, Liu G, et al. (2010) Effects of estrogen on breast cancer development: role of estrogen receptor independent mechanisms. Int $J$ Cancer 127: 1748-1757.

22. Hamidullah P, Changkija B, Konwar R (2012) Role of interleukin-10 in breast cancer. Breast Cancer Res Treat 133: 11-21. 
Citation: Hassan Z (2019) Anti-cancer and Biotherapeutic Potentials of Probiotic Bacteria. J Cancer Sci Ther 11: 009-013. doi: 10.4172/19485956.1000575

23. Sheikhpour E, Noorbakhsh P, Foroughi E (2018) A survey on the role of interleukin-10 in breast cancer: A narrative. Rep Biochem Mol Biol 7: 30-37.

24. Hasegawa T, Kanasugi H, Hidaka M, Yamamoto T, Abe S, et al. (1996) Effect of orally administered heat-killed Enterococcus faecalis FK-23 preparation on neutropenia in dogs treated with cyclophosphamide. Int J Immunopharmacol 18: 103-112.

25. Veiga P, Gallini CA, Beal C, Michaud M, Delaney ML, et al. (2010) Bifidobacterium animalis subsp. Lactis fermented milk product reduces inflammation by altering a niche for colitogenic microbes. Proc Natl Acad Sci USA 107: 18132-18137.

26. Gaudier E, Michel C, Segain J, Cherbut C, Hoebler C (2005) The VSL\# 3 probiotic mixture modifies microflora but does not heal chronic dextran-sodium sulfate-induced colitis or reinforce the mucus barrier in mice. J Nutr 135: $2753-$ 2761.

27. Pertea G, Huang X, Liang F, Antonescu V, Sultana R, et al. (2003) TIGR gene indices clustering tools (TGICL): A software system for fast clustering of large EST datasets. Bioinformatics 19: 651-652.

28. Rafter J (2003) Probiotics and colon cancer. Best Pract Res Clin Gastroentero 17: 849-859.

29. O'Mahony L, Feeney M, O'Halloran S, Murphy L, Kiely B, et al. (2001). Probiotic impact on microbial flora, inflammation and tumour development in IL-10 knockout mice. Aliment Pharmacol Ther 15: 1219-1225.

30. Malhotra SL (1977) Dietary factors in a study of colon cancer from cance registry, with special reference to the role of saliva, milk and fermented milk products and vegetable fibre. Med Hypotheses 3: 122-134.

31. Lidbeck A, Overvikf E, Rafter J, Nord CE, Gustafssonf J (1992) Effect of Lactobacillus acidophilus supplements on mutagen excretion in faeces and urine in humans. Microb Ecol Health Dis 5: 59-67.

32. Van't Veer P, Dekker JM, Lamers JW, Kok FJ, Schouten EG, et al. (1989) Consumption of fermented milk products and breast cancer: A case-contro study in the Netherlands. Cancer Res 49: 4020-4023.

33. Toi M, Hirota S, Tomotaki A, Sato N, Hozumi Y, et al. (2013) Probiotic beverage with soy isoflavone consumption for breast cancer prevention: A case-control study. Curr Nutr Food Sci 9: 194-200.

34. Peters RK, Pike MC, Garabrant D, Mack TM (1992) Diet and colon cancer in Los Angeles County, California. Cancer Causes Control 3: 457-473.

35. Young TB, Wolf DA (1988) Case-control study of proximal and distal colon cancer and diet in Wisconsin. Int J Cancer 42: 167-175.

36. Sobhani I, Amiot A, Le Baleur Y, Levy M, Auriault ML, et al. (2013) Microbia dysbiosis and colon carcinogenesis: Could colon cancer be considered a bacteria-related disease?. Therap Adv Gastroenterol 6: 215-229.

37. Zhu Q, Gao R, Wu W, Qin H (2013) The role of gut microbiota in the pathogenesis of colorectal cancer. Tumor Biol 34: 1285-1300.

38. Johansson ML, Nobaek S, Berggren A, Nyman M, Bjorck I, et al. (1998) Survival of Lactobacillus plantarum DSM 9843 (299v), and effect on the short-chain fatty acid content of faeces after ingestion of a rose-hip drink with fermented oats. Int J Food Microbiol 42: 29-38.

39. Ayebo A, Angelo I, Shahani K (1980) Effect of ingesting Lactobacillus acidophilus milk upon fecal flora and enzyme activity in humans. Milchwissenschaft 35: 730-733

40. Zhang G, Zhang J, Wang X, Yang W, Sun Z, et al. (2014) Apoptosis of human tongue sqamous cell carcinoma cell (CAL-27) induced by Lactobacillus. J Appl Oral Sci 22: 282-286

41. Mego M, Holec V, Drgona L, Hainova K, Ciernikova S, et al. (2013) Probiotic bacteria in cancer patients undergoing chemotherapy and radiation therapy. Complement Ther Med 21: 712-723.

42. Di Francia R, Siesto RS, Valente D, Del Buono A, Pugliese S, et al. (2013) Current strategies to minimize toxicity of oxaliplatin: Selection of pharmacogenomic panel tests. Anticancer Drugs 2: 1-11.
43. Gradishar W J, Vokes EE (1990) Review 5-Fluorouracil cardiotoxicity: A critica review. Ann Oncol 1: 409-414.

44. Soares PM, Mota JM, Gomes AS, Oliveira RB, Assreuy AM, et al. (2008) Gastrointestinal dysmotility in 5-fluorouracil-induced intestinal mucositis outlasts inflammatory process resolution. Cancer Chemother Pharmacol 63 : 91-98.

45. Duncan M, Grant G (2003) Oral and intestinal mucositis-causes and possible treatments. Aliment Pharmacol Ther 18: 853-874.

46. Smith CL, Geier MS, Yazbeck R, Torres DM, Butler RN, et al. (2008) Lactobacillus fermentum BR11 and fructo-oligosaccharide partially reduce jejunal inflammation in a model of intestinal mucositis in rats. Nutr Cancer 60 : 757-767.

47. Whitford EJ, Cummins AG, Butler RN, Prisciandaro LD, Fauser JK, et al (2009) Effects of Streptococcus thermophilus $\mathrm{TH}-4$ on intestinal mucositis induced by the chemotherapeutic agent, 5-Fluorouracil (5-FU). Cancer Biol Ther 8: 505511.

48. Justino PF, Melo LF, Nogueira AF, Morais CM, Mendes WO, et al. (2015) Regulatory role of Lactobacillus acidophilus on inflammation and gastric dysmotility in intestinal mucositis induced by 5 -fluorouracil in mice. Cancer Chemother Pharmacol 75: 559-567.

49. Mack RD, Lebel S (2004) Role of probiotics in the modulation of intestinal infections and inflammation. Curr Opin Gastroenterol 20: 22-26.

50. Chitapanarux I, Chitapanarux T, Traisathit P, Kudumpee S, Tharavichitku E, et al. (2010) Randomized controlled trial of live Lactobacillus acidophilus plus bifidobacterium bifidum in prophylaxis of diarrhea during radiotherapy in cervical cancer patients. Radiat Oncol 5: 31.

51. Mego M, Koncekova R, Mikuskova E, Drgona L, Ebringer L, et al. (2006) Prevention of febrile neutropenia in cancer patients by probiotic strain Enterococcus faecium M-74. Phase II study. Support Care Cancer 14: 285-290.

52. Bowen JM, Stringer AM, Gibson RJ, Yeoh AS, Hannam S, et al. (2007) VSL\#3 probiotic treatment reduces chemotherapy-induced diarrhea and weight loss. Cancer Biol Ther 6: 1449-1454.

53. Takeda K, Okumura K (2007) Effects of a fermented milk drink containing Lactobacillus casei strain shirota on the human NK-cell activity. J Nutr 137: 791-793.

54. Arimochi H, Kinouchi T, Kataoka K, Kuwahara T, Ohnishi Y (1997) Effect of intestinal bacteria on formation of azoxymethane-induced aberrant crypt foci in the rat colon. Biochem Biophys Res Commun 238: 753-757.

55. Goldin BR, Gualtieri LJ, Moore RP (1996) The effect of Lactobacillus GG on the initiation and promotion of $\mathrm{DMH}$-induced intestinal tumors in the rat. Nutr Cancer 25: 197-204.

56. Donato KA, Gareau MG, Wang YJ, Sherman PM (2010) Lactobacillus rhamnosus GG attenuates interferon- $y$ and tumour necrosis factor-alphainduced barrier dysfunction and pro-inflammatory signalling. Microbiology 156: 3288-3297.

57. Logan RM, Stringer AM, Bowen JM, Yeoh AS, Gibson RJ, et al. (2007) The role of pro-inflammatory cytokines in cancer treatment-induced alimentary tract mucositis: Pathobiology, animal models and cytotoxic drugs. Cancer Treat Rev 33: 448-460.

58. Liong MT (2008) Roles of probiotics and prebiotics in colon cancer prevention postulated mechanisms and in-vivo evidence. Int J Mol Sci 9: 854-863.

59. Liu TZ, Chen CY, Yiin SJ, Chen CH, Cheng JT (2006) Molecular mechanism of cell cycle blockage of hepatoma SK-Hep-1 cells by Epimedin C through suppression of mitogen-activated protein kinase activation and increased expression of CDK inhibitors p21 Cip1 and p27 Kip1. Food Chem Toxicol 44 227-235.

60. Terai S, Okita K (1999) Does cytoplasmic cyclin D1 regulate the cell cycle directly?. J Gastroenterol 34: 547-548. 